\title{
Stroke in Patients with Diabetes: Is It Time to Expand Public Health Priority to Encompass High-Risk Patients with Increased Insulin Resistance?
}

\author{
Authors: \\ Sian Alexandra Bradley, ${ }^{1,2}$ Francis Muttamthottil Varghese, ${ }^{3}$ Bindu \\ Menon, ${ }^{4}$ Man Mohan Mehndiratta, ${ }^{5}$ *Sonu Menachem Maimonides \\ Bhaskar ${ }^{1,2,6,7,8}$ \\ 1. Neurovascular Imaging Laboratory, Ingham Institute for Applied Medical \\ Research, Clinical Sciences Stream, Sydney, Australia \\ 2. South Western Sydney Clinical School, University of New South Wales (UNSW), \\ Sydney, Australia \\ 3. Department of Headache and Neuro-ophthalmology, Teresa Eye and Migraine \\ Centre, Cherthala, Kerala, India \\ 4. Department of Neurology, Apollo Hospitals, Nellore, India \\ 5. Department of Neurology, BLK Super Speciality Hospital, New Delhi, India \\ 6. Department of Neurology and Neurophysiology, Liverpool Hospital and South \\ Western Sydney Local Health District (SWSLHD), Sydney, Australia \\ 7. NSW Brain Clot Bank, NSW Health Statewide Biobank and NSW Health \\ Pathology, Sydney, Australia \\ 8. Stroke and Neurology Research Group, Ingham Institute for Applied Medical \\ Research, Sydney, Australia \\ *Correspondence to sonu.bhaskar@reprogramglobal.org/sonu.bhaskar@uon.edu.au
}

Disclosure:

The authors have declared no conflicts of interest.

Acknowledgements: Funding for the NSW Brain Clot Bank (Chief Investigator: Sonu Menachem Maimonides Bhaskar) from the NSW Ministry of Health (2019-2022) is acknowledged. The funding body had no role in the study design, data collection, analysis, interpretation of findings, or manuscript preparation. The content is solely the responsibility of the authors and does not necessarily represent the official views of the affiliated/funding organisation(s).

Received:

07.08 .21

Accepted:

17.08.21

Keywords:

Cerebrovascular disease, diabetes, global health, insulin resistance, public health, stroke.

Citation:

EMJ Diabet. 2021;9[1]:84-91.

\begin{abstract}
Diabetes and stroke, with an interlinking aetiology, contribute to a growing cardiovascular disease burden and mortality around the world. Given the disproportionate prevalence and the burden of these conditions in the developing world, as well as the high risk of both Type 2 diabetes and cardiovascular disease carried by patients with metabolic syndrome, public health strategies are vital to mitigate the impact. Systematic approaches towards identifying undiagnosed patients in the community and building health systems around those targeted interventions have been implemented. However, growing evidence indicates potential for approaches to capture high-risk patients, such as those who suffer from pre-diabetes or increased insulin resistance, to provide early and optimal treatments, which could translate to population-level benefits, including reduced prevalence, disability, and disease burden.
\end{abstract}




\section{INTRODUCTION}

Cardiovascular disease (CVD), including stroke, is a leading cause of illness, death, and disability worldwide. $\quad$ A predominant factor driving morbidity and mortality is diabetes, which is a major contributor to poor outcomes in acute ischaemic stroke. ${ }^{2,3}$ Given the increasing prevalence of diabetes in the world and more specifically in Asia, ${ }^{4,5}$ it is warranted that an approach directed towards understanding the trajectories of patients with diabetes experiencing a stroke or those at a high risk of diabetes needs to be addressed. In this article, the authors discuss the emerging burden and associated morbidity of stroke in patients with diabetes and provide insights on therapeutic management from a public health perspective. The authors also provide insights on shifting the public health message towards identifying patients in the community at high risk of developing diabetes or stroke or both.

\section{STROKE IN PATIENTS WITH DIABETES}

Diabetes puts patients at higher risk for cardiovascular events, including fatal stroke, non-fatal stroke, and transient ischaemic attack. ${ }^{6}$ The clinical profile of diabetic stroke differs from a non-diabetic stroke. ${ }^{7}$ A Cochrane review showed that concurrent metabolic syndrome and diabetes were associated with recurrent strokes in patients with minor stroke and transient ischaemic attack. ${ }^{8,9}$ Furthermore, a diagnosis of diabetes is associated with an increased risk of lacunar strokes, which are associated with stroke recurrence. ${ }^{13}$ There is also evidence to suggest an increased burden of post-stroke poor clinical outcomes, such as functional disability, in patients with diabetes relative to patients without diabetes, particularly those with admission hyperglycaemia., 11,12 Diabetes is associated with greater mortality, poorer neurological outcomes, poorer stroke rehabilitation outcomes, and futile recanalisation. ${ }^{13-17}$ The overall disease, financial, and social burden of stroke and diabetes is provided in Table 1.

It has been suggested that HbAlc levels are associated with the development of cerebral white matter damage, as higher levels of white matter damage have been seen in patients with diabetic stroke. 10,28 There is conflicting evidence over whether a history of diabetes predisposes a patient to dementia, including Alzheimer's disease. ${ }^{29,30}$ A 2016 large sample cohort study found a significant association between a history of diabetes and brain infarction, particularly lacunar strokes, with a lower mini-mental state examination (MMSE) score at the end of life, but not with Alzheimer's disease generally.13 A novel 2021 mouse model of post-stroke cognitive impairment showed a new biomarker of brain and serum quinolinic acid concentrations and quinolinic acid-to-kynurenic acid ratios that was increased in diabetic mice. ${ }^{14}$ It was associated with long-term memory impairment, leukoaraiosis, neuronal death, and microglial and macrophage infiltration. Given the increasing burden of diabetes and global ageing populations, the impact of diabetes on cognitive function is an area of concern.

There is a lack of defined treatment protocols targeted at patients with diabetes and stroke. Given the different clinical profiles of stroke in patients with diabetes relative to patients without diabetes, there is a necessity for collaborative management between primary healthcare physicians, endocrinologists, internal medicine physicians, and neurologists. Whilst some guidelines recommend against the use of tissue plasminogen activator in patients with diabetes and previous stroke, recent evidence has shown that tissue plasminogen activator is beneficial for use in such patients. ${ }^{15-17}$ There is also a lack of consensus about the use of glucose control in stroke treatment for patients with diabetes. A 2020 meta-analysis recommended against the use of tight glucose control after ischaemic stroke because it does not improve neurological or functional outcomes but rather increases the risk of symptomatic intracranial haemorrhage and hypoglycaemia. ${ }^{31}$ 
Table 1: The burden of disease, prevalence, disability-adjusted life years, and financial burden due to diabetes, stroke, diabetic stroke, and pre-diabetes.

\begin{tabular}{|c|c|c|c|c|}
\hline & Burden of disease & Prevalence & DALY & Financial burden \\
\hline Diabetes & $\begin{array}{l}\text { 9.30\% or } 463 \text { million } \\
\text { people (global } \\
\text { prevalence in 2019) }{ }^{28}\end{array}$ & N/R & $\begin{array}{l}2.8(\% \text { of DALYs } \\
2019)^{19}\end{array}$ & $\begin{array}{l}827 \text { billion USD } \\
\text { (direct medical } \\
\text { costs) } \\
1.7 \text { trillion USD } \\
\text { (Loss in GDP } \\
\text { estimate from } \\
2011-2013)^{20}\end{array}$ \\
\hline Stroke & $\begin{array}{l}42.43 \text { million } \\
\text { (prevalence of } \\
\text { cerebrovascular } \\
\text { disease in 2015) } \\
\\
13.70 \text { million people } \\
(\text { global incidence in } \\
2016)^{22}\end{array}$ & $\mathrm{~N} / \mathrm{R}$ & $\begin{array}{l}5.7(\% \text { of DALYs } \\
2019)^{19}\end{array}$ & $\begin{array}{l}\text { Global statistics } \\
\text { couldn't be found } \\
\text { In Australia: } \\
\$ 32.2 \text { billion lost } \\
(2020)^{23}\end{array}$ \\
\hline $\begin{array}{l}\text { Diabetic } \\
\text { stroke }\end{array}$ & $\begin{array}{l}65 \% \text { (proportion of } \\
\text { deaths caused by } \\
\text { diabetes that are } \\
\text { attributable to CVD or } \\
\text { stroke or both in the } \\
\text { USA) }\end{array}$ & $\begin{array}{l}3.84 \text { million } \\
\text { (prevalence of } \\
\text { diabetes in stroke } \\
\text { is estimated to be } \\
28 \%{ }^{24} \text { Twenty- } \\
\text { eight percent } \\
\text { multiplied by } 13.70 \\
\text { million in } 2016 \text { ) }\end{array}$ & $\begin{array}{l}32.48 \text { million } \\
\text { DALYs lost ( } 28 \% \\
\text { multiplied by } 116 \\
\text { million DALYs lost } \\
\text { in 2016) }\end{array}$ & $\begin{array}{l}\text { The median } \\
\text { annual costs per } \\
\text { patient for stroke } \\
\text { are approximately } \\
322 \% \text { higher } \\
\text { compared with } \\
\text { those for patients } \\
\text { with T2DM but } \\
\text { without CVD } 26\end{array}$ \\
\hline Pre-diabetes & $\begin{array}{l}352.1 \text { million ( } 7.3 \% \text { ) } \\
\text { (prevalence of } \\
\text { impaired glucose } \\
\text { tolerance in 2017) } 27\end{array}$ & $N / R$ & $N / R$ & $\begin{array}{l}\$ 43.4 \text { billion for } \\
\text { pre-diabetes } 2017 \\
\text { (the USA only) }^{9}\end{array}$ \\
\hline
\end{tabular}

CVD: cardiovascular disease; DALY: disability-adjusted life years; GDP: gross domestic product; N/R: not reported; T2DM: Type 2 diabetes mellitus.

\section{PRE-DIABETES}

Early identification of patients with prediabetes is important to reduce the burden of comorbid diabetes and stroke. A 2017 prospective cohort study compared pre-diabetes definitions that used fasting glucose (American Diabetes Association [ADA] and World Health Organization [WHO]), $\mathrm{HbA1c}$ (ADA and International Expert Committee [IEC]), and the 2-hour glucose tolerance test (ADA and WHO).

It found that HbA1c pre-diabetes definitions were more specific and provided a better indication of clinical complication risk. ${ }^{32}$ However, there is concern that some patients classified as prediabetic from glucose testing may be determined to be normoglycaemic when $\mathrm{HbAlc}$ alone is used, particularly patients with impaired fasting glucose. ${ }^{33}$ Furthermore, HbA1c is only an indirect measure of insulin resistance. There are also issues of availability of $\mathrm{HbA1c}$ testing at primary healthcare practices, particularly in certain Asian, African, and South American regions. ${ }^{34}$ Capturing 
at-risk individuals earlier would reduce the risk of complications associated with diabetes, and the homeostatic model assessment of insulin resistance (HOMA-IR) is a rarely used tool that can indicate insulin sensitivity and $\beta$-cell function prior to an increase in glucose levels. ${ }^{35}$

\section{PUBLIC HEALTH APPROACHES AVAILABLE IN TARGETING THIS POPULATION}

Public health approaches towards populationlevel screening and prevention of diabetes and stroke vary across countries. A list of public health strategies implemented in several nations is provided in Table 2.

\section{Role of Primary Healthcare Networks}

The role of primary healthcare networks is critical in the early capture and routine monitoring of at-risk patients. Patients should receive serial glucose monitoring and should be categorised into high, moderate, and low-risk groups to determine the necessity for prophylactic antidiabetic medication and lifestyle management. In Australia, the National Association of Diabetes Centres (NADC) network membership allows primary, secondary, and tertiary level care facilities to collaborate towards better outcomes for patients with diabetes. ${ }^{3}$ In the UK, the Diabetes UK Primary Care Network provides monthly newsletters, expert information, and resources to practitioners. ${ }^{4}$ In India, the National Programme on Prevention and Control of Cancer, Diabetes, Cardiovascular Diseases and Stroke (NPCDCS), which was launched in 2010, utilises opportunistic screening for hypertension and diabetes for persons above 30 years. ${ }^{37}$ The programme functions at all four levels of healthcare: village, sub-centre, community health centres, and district hospitals. ${ }^{44}$

Table 2: Country-level public health strategies in diabetes screening and prevention

\begin{tabular}{|c|c|}
\hline Country & Public health strategy \\
\hline USA & The Centers for Disease Control and Prevention (CDC) prediabetes risk test. ${ }^{36}$ \\
\hline India & $\begin{array}{l}\text { National Programme for Prevention and Control of Cancer, Diabetes, } \\
\text { Cardiovascular Diseases and Stroke (NPCDCS) focuses on screening of the } \\
\text { common non-communicable diseases, at sub-centres, primary health centres, } \\
\text { district, and above, through the setting up of non-communicable disease } \\
\text { clinics. }{ }^{37}\end{array}$ \\
\hline China & $\begin{array}{l}65 \% \text { (proportion of deaths caused by diabetes that are attributable to CVD or } \\
\text { stroke or both in the USA) }\end{array}$ \\
\hline Singapore & 352.1 million (7.3\%) (prevalence of impaired glucose tolerance in 2017) ${ }^{27}$ \\
\hline New Zealand & Pre-diabetes and self-management pilot projects. ${ }^{40}$ \\
\hline Australia & $\begin{array}{l}\text { Reduce modifiable risk factors in the general population (physical activity, } \\
\text { healthy eating, education and social media campaigns, healthy food } \\
\text { availability, upskill primary healthcare physicians and public health workforce } \\
\text { to support patients, especially Aboriginal and Torres Strait Islanders. }{ }^{41} \\
\text { AUSDRISK tool. }{ }^{42}\end{array}$ \\
\hline UK & $\begin{array}{l}\text { National Health Services Diabetes Prevention Programme ([NHS DPP] identify } \\
\text { high-risk individuals and refer them to a behaviour change programme). }{ }^{43}\end{array}$ \\
\hline
\end{tabular}

AUSDRISK: Australian Type 2 Diabetes Risk Assessment Tool. 
Moreover, other community-led initiatives, such as a network of volunteer female community health workers, envisaged to link vulnerable individuals with the public health sector, government health initiatives, and national health programmes. ${ }^{18}$ Limited funding and underutilisation are major concerns. Mexico's national Integrated Management of Diabetes in Stages programme at Mexico's Institute for Social Security and Services for State Workers clinics promoted patient empowerment and education nationwide through outpatient consultations with a multidisciplinary team from 2007-2014.19 An analysis of the programme determined it to be a feasible and suitable programme to address this issue. ${ }^{20}$

\section{Social Prescribing}

Social prescribing (SP) is a method used by primary healthcare practitioners, initially implemented in the UK, that aims to connect patients with local and social services through prescription to improve patient wellbeing and health in a variety of areas. ${ }^{45}$ A review of SP practices in Bristol, UK, described three different models: SP Light, SP Medium, and SP Holistic. SP Holistic projects aim to play a preventative role and work to improve long-term conditions. ${ }^{46}$ They involve partnerships between general practitioners and tertiary care partners and sometimes evolve from SP Light and Medium models. SP Light projects aim to refer at-risk patients to specific programmes to improve outcomes, for example, prescribing exercise to a patient. In SP Medium, a health facilitator will see referred patients and provide advice on exercise, diet, programmes, and mental health support among other resources. ${ }^{46}$

An evaluation of the Community Connectors Social Prescribing Service in Bradford, UK, reported increasing coverage of the population and general practice clinics and found overall improvements of health, mental wellbeing, and social connectedness. ${ }^{47}$ An evidence synthesis of available UK studies on SP, however, found that there is not enough evidence to support the effectiveness of such programmes given the lack of uniformity in how practices implement such programmes and the need for more largescale studies on their impact.48 SP is gaining attention internationally, and a joint report by the Royal Australian College of General Practitioners
(RACGP) and the Consumers Health Forum (CHF) recommended the implementation within the Australian healthcare system to incorporate local, non-clinical services into primary care. ${ }^{49}$ Despite emerging benefits, high quality and comparable evaluations of the efficacy and costeffectiveness of SP as a public health intervention are warranted. ${ }^{50}$

\section{Undiagnosed Diabetes}

Undiagnosed diabetes is a major problem in the community, and methods to improve asymptomatic diagnosis are needed. Epidemiological studies have demonstrated the link between insulin resistance and CVD risk, including for stroke.51,52 The landmark Insulin Resistance Intervention after Stroke (IRIS) trial showed that in insulin-resistant patients $(n=3,876)$ without diabetes who had a recent ischaemic stroke or transient ischaemic attack, pioglitazone, a glucose-lowering drug (thiazolidinediones), reduced the risk of future vascular events, such as stroke or myocardial infarction, by $24 \%$, and progression to diabetes by $52 \%$, relative to those who received placebo.53 This, along with secondary analyses of IRIS data, ${ }^{54}$ highlighted the preferential use of pioglitazone in high-risk patients for stroke with Type 2 diabetes mellitus (T2DM) for primary and secondary stroke prevention. ${ }^{54,55}$ More recently, during the COVID-19 pandemic, metformin use has been associated with reduced mortality and severity in patients with T2DM. ${ }^{56}$ However, close monitoring for lactic acidosis and deterioration of kidney function is needed in patients with severe COVID-19. Moreover, population-based studies have also indicated association of increased insulin resistance, metabolic syndrome, and diabetes with increased levels of inflammatory markers, such as C-reactive protein, IL-6, and TNF-a. ${ }^{57,58}$ Use of such inflammatory biomarkers could also be considered as a useful strategy in screening patients at high risk for CVD. This is especially a problem in under-resourced settings, due to lack of access to primary care physicians, appropriate tests, and immaturity of primary healthcare networks. ${ }^{59,60}$ South Asian migrants to highly developed countries such as the UK are at a particularly high risk of CVD, due to a combination of systemic disparities and structural and innate factors. This group is a target group due to their higher risk of 
developing T2DM. ${ }^{61}$ Similarly, Canadian studies have shown that migrants from South Asia, SubSaharan Africa, Latin America, and the Caribbean have a higher burden of diabetes and CVD. ${ }^{62} \mathrm{~A}$ recent study also showed that the prevalence of undiagnosed pre-diabetes in migrant workers in Singapore increased their risk of pneumonia and electrolyte abnormalities from COVID-19.63

Recent population studies have indicated a role of retinopathy in screening high-risk individuals (e.g., those who are overweight or have elevated fasting glucose or impaired glucose tolerance) for pre-diabetes. ${ }^{64-67}$ Given the wide availability of fundus photography, as well as the ease of delineating retinal lesions, this strategy could be useful; however, this needs further validation and targeted studies in high-risk patients to warrant public health indication. ${ }^{68}$ Moreover, an important consideration is the sensitivity of the ophthalmoscopic method. This is because a prevalence of retinal lesions as high as $9.8 \%$ using 6 -field fundus photography was reported in the Blue Mountain Study, ${ }^{69}$ in contrast to $<1 \%$ in the Framingham Eye Study ${ }^{70}$ and Göteborg ${ }^{71}$ study in non-diabetic populations.

Community education and partnership in identifying patients at high risk of diabetes are needed, as well as awareness of diabetes in these groups. The development of targeted education modules for demonstration programmes targeted at the populations in their own languages would be beneficial. Furthermore, building the capacity of local community health workers that can teach patients about risk factors, take blood samples, participate in community-based surveillance, and utilise questionnaires to indicate symptomsbased or self-reported diabetes should be the main priority. Also, an increased awareness about post-stroke rehabilitation in patients with diabetes is needed, as these patients should be proactively educated about the risk of secondary stroke. ${ }^{72,73}$

\section{CONCLUSION}

There is an increasing need for improving surveillance of diabetes in the community and among primary healthcare networks because of the elevated risks associated with stroke in this subgroup of patients. The proposed shift towards population-based strategies (from patients with T2DM to those with pre-diabetes, metabolic syndrome, or insulin resistance), as well as using preventive measures for high-risk patients, could allow detection of those who are at increased risk of having a stroke. This approach would be helpful to improve the quality of life and reduce the burden of these chronic cardio-metabolic diseases. Furthermore, in the COVID-19 era, given the known risks of increased susceptibility to COVID-19 infections among patients with diabetes or pre-diabetes, as well as their poor clinical outcomes and increased mortality, it is imperative to explore alternative strategies to promptly identify patients at high risk, in order to maximise the opportunity to mitigate the ongoing impact of this devastating pandemic. ${ }^{74-76}$ Strategies should also focus on disproportionate burden on vulnerable communities and those from low-resource settings. ${ }^{75}$

\section{References}

1. Roth GA et al. Global burden of cardiovascular diseases and risk factors, 1990-2019: update from the GBD 2019 study. J Am Coll Cardiol. 2020;76(25):2982-3021.

2. Desilles JP et al. Diabetes mellitus, admission glucose, and outcomes after stroke thrombolysis: a registry and systematic review. Stroke. 2013;44(7):1915-23.

3. Lu GD et al. Effects of diabetes mellitus and admission glucose in patients receiving mechanical thrombectomy: a systematic review and meta-analysis. Neurocrit Care. 2018;9(3):426-34.
4. Nanditha A et al. Diabetes in Asia and the Pacific: implications for the global epidemic. Diabetes Care. 2016;39(3):472-85.

5. Yap $\mathrm{J}$ et al. Impact of type 2 diabetes and microvascular complications on mortality and cardiovascular outcomes in a multiethnic Asian population. BMJ Open Diabetes Res Care. 2021;9(1):e001413.

6. The EZ et al. Long-term outcomes of ischaemic stroke patients with diabetes in a multi-ethnic cohort in Singapore. Ann Acad Med Singap. 2021;50(1):16-25.
7. Bhaskar S. Impact of obesityinduced type 2 diabetes on longterm outcomes following stroke. Clin Sci (Lond). 2019;133(14):1603-7.

8. Chen $\mathrm{R}$ et al. Diabetes and stroke: epidemiology, pathophysiology, pharmaceuticals and outcomes. Am J Med Sci. 2016;351(4):380-6.

9. Chen $\mathrm{W}$ et al. Recurrent stroke in minor ischemic stroke or transient ischemic attack with metabolic syndrome and/or diabetes mellitus. J Am Heart Assoc. 2017;6(6):e005446.

10. Rastogi A et al. Emerging 
role of white matter lesions in cerebrovascular disease. Eur J Neurosci. 2021;54(4):5531-59.

11. Fang $\mathrm{HJ}$ et al. Prognostic value of admission hyperglycemia on outcomes of thrombolysis in ischemic stroke patients with or without diabetes. Chin Med J (Engl). 2020;133(18):2244-6.

12. Tsivgoulis $\mathrm{G}$ et al. Association of baseline hyperglycemia with outcomes of patients with and without diabetes with acute ischemic stroke treated with intravenous thrombolysis: a propensity scorematched analysis from the SITS-ISTR Registry. Diabetes. 2019;68:1861-9.

13. Abner EL et al. Diabetes is associated with cerebrovascular but not Alzheimer's disease neuropathology. Alzheimers Dement. 2016;12(8):882-9.

14. Cogo A et al. Increased serum QUIN/ KYNA is a reliable biomarker of post-stroke cognitive decline. Mol Neurodegener. 2021;16(1):7.

15. Fuentes B et al. Acute ischemic stroke patients with diabetes should not be excluded from intravenous thrombolysis. J Thromb Thrombolysis. 2014;38(4):522-7.

16. Powers WJ et al. Guidelines for the early management of patients with acute ischemic stroke: 2019 update to the 2018 guidelines for the early management of acute ischemic stroke: a guideline for healthcare professionals from the American Heart Association/ American Stroke Association. Stroke. 2019;50(12):e344-e418.

17. Ehrlich ME et al. Intravenous tissuetype plasminogen activator in acute ischemic stroke patients with history of stroke plus diabetes mellitus. Stroke. 2019;50(6):1497-503.

18. Saeedi $P$ et al. Global and regional diabetes prevalence estimates for 2019 and projections for 2030 and 2045: results from the International Diabetes Federation Diabetes Atlas, 9th edition. Diabetes Res Clin Pract. 2019;157:107843.

19. GBD 2019 Diseases and Injuries Collaborators. Global burden of 369 diseases and injuries in 204 countries and territories, 1990-2019: a systematic analysis for the Global Burden of Disease study 2019. Lancet. 2020;396(10258):1204-22.

20. World Health Organization (WHO). Global report on diabetes 2016. 2016. Available at: https://apps. who.int/iris/bitstream/handle/ 10665/204871/9789241565257_eng. pdf. Last accessed: 7 August 2021.

21. Roth GA et al. Global, regional, and national burden of cardiovascular diseases for 10 causes, 1990 to 2015 . J Am Coll Cardiol. 2017;70(1):1-25

22. World Stroke Organization (WSO). Global stroke fact sheet. 2017. Available at: https://www.world-
stroke.org/assets/downloads/WSO Global_Stroke_Fact Sheet.pdf.Last accessed: 7 August 2021.

23. Deloitte Access Economics. The economic impact of stroke in Australia, 2020. 2020. Available at: https://www2.deloitte.com/ content/dam/Deloitte/au/ Documents/Economics/deloitteau-dae-economic-impact-strokereport-061120.pdf. Last accessed: 7 August 2021

24. Lau LH et al. Prevalence of diabetes and its effects on stroke outcomes: a meta-analysis and literature review. J Diabetes Investig. 2019;10(3):780-92.

25. GBD 2016 Stroke Collaborators. Global, regional, and national burden of stroke, 1990-2016: a systematic analysis for the Global Burden of Disease study 2016. Lancet Neurol. 2019:18(5):439-58.

26. Einarson TR et al. Economic burden of cardiovascular disease in type 2 diabetes: a systematic review. Value Health. 2018;21(7):881-90

27. International Diabetes Federation (IDF). IDF Diabetes Atlas 9th edition. 2019. Available at: https:// diabetesatlas.org/en/. Last accessed: 7 August 2021

28. Rozanski M et al. Elevated levels of hemoglobin A1c are associated with cerebral white matter disease in patients with stroke. Stroke. 2014;45(4):1007-11.

29. Cumming TB, Brodtmann A. Can stroke cause neurodegenerative dementia? Int J Stroke. 2011;6(5):416-24.

30. Sherzai D et al. The association between diabetes and dementia among elderly individuals: a nationwide inpatient sample analysis. J Geriatr Psychiatry Neurol. 2016;29(3):120-5.

31. Cerecedo-Lopez CD et al. Insulin in the management of acute ischemic stroke: a systematic review and meta-analysis. World Neurosurg. 2020;136:514-34

32. Warren B et al. Comparative prognostic performance of definitions of prediabetes: a prospective cohort analysis of the Atherosclerosis Risk in Communities (ARIC) study. Lancet Diabetes Endocrinol. 2017:5(1):34-42.

33. Bergman $\mathbf{M}$ et al. Diabetes prevention: global health policy and perspectives from the ground. Diabetes Manag (Lond). 2012;2(4):309-21.

34. World Health Organization (WHO). General availability of diabetes testing (by blood glucose measurement, OGTT) at the primary health care level. 2021. Available at: https://www.who.int/data/gho/ data/indicators/indicator-details/ $\mathrm{GHO} /$ general-availability-ofdiabetes-testing-(by-blood-glucosemeasurement-ogtt)-at-the-primaryhealth-care-level. Last accessed: 7
August 2021

35. Wallace TM et al. Use and abuse of HOMA modeling. Diabetes Care. 2004;27(6):1487-95.

36. Centers for Disease Control and Prevention (CDC). Prediabetes risk test. 2019. Available at: https:// www.cdc.gov/prediabetes/pdf/ Prediabetes-Risk-Test-Final.pdf. Last accessed: 7 August 2021.

37. Ministry of Health and Family Welfare, Government Of India. National Programme for Prevention and Control of Cancer, Diabetes, Cardiovascular Diseases \& Stroke (NPCDCS) operational guidelines (revised: 2013-17). 2017. Available at: https://main.mohfw.gov.in/ sites/default/files/Operational\%20 Guidelines\%20of\%2ONPCDCS\%20 \%28Revised\%20-\%202013-17\%29 1. pdf. Last accessed: 7 August 2021.

38. Luo $Z$ et al. Meeting the challenge of diabetes in China. Int $\mathrm{J}$ Health Policy Manag. 2020;9(2):47-52.

39. Ministry of Health, Singapore. Ministry of Health studying measures to better support persons with pre-diabetes. 2017. Available at: https://www.moh. gov.sg/news-highlights/details/ ministry-of-health-studying-measuresto-better-support-persons-with-prediabetes\#: :text=The\%20ACG\%20 equips $\% 20$ clinicians $\% 20$ with,for $\% 20$ effecting $\% 20$ sustained $\% 20$ behavioural\%20changes. Last accessed: 7 August 2021.

40. Ministry of Health, New Zealand. Pre-diabetes and self-management of long term conditions. 2016. Available at: https://www.health govt.nz/our-work/diseases-andconditions/diabetes/about-diabetes/ pre-diabetes-and-self-managementlong-term-conditions. Last accessed: 7 August 2021.

41. Australian Government, Department of Health. Australian National Diabetes Strategy 2016-2020. 2015. Available at: https://www. health.gov.au/sites/default/ files/documents/2019/09/ australian-national-diabetesstrategy-2016-2020_1.pdf. Last accessed: 7 August 2021.

42. Australian Government, Department of Health. The Australian Type 2 Diabetes Risk Assessment Tool (AUSDRISK). 2020. Available at: https://www.health gov.au/resources/apps-and-tools/ the-australian-type-2-diabetesrisk-assessment-tool-ausdrisk. Last accessed: 7 August 2021.

43. NHS. NHS Diabetes Prevention Programme (NHS DPP). Available at: https://www.england.nhs.uk/diabetes/ diabetes-prevention/. Last accessed: 7 August 2021

44. Raghuveer $P$ et al. Opportunistic screening for diabetes mellitus and hypertension in primary care settings in Karnataka, India: a few steps forward but still some way to go. 
F1000Res. 2020;9:335.

45. Pescheny JV et al. The impact of social prescribing services on service users: a systematic review of the evidence. Eur J Public Health. 2020;30(4):664-73.

46. Kimberlee RH. Developing a social prescribing approach for Bristol. 2013. Available at: https://uwe-repository. worktribe.com/output/927254. Last accessed: 7 August 2021

47. Dayson C, Leather D. Evaluation of HALE Community Connectors Social Prescribing Service 2018-19. 2020. Available at: http://shura.shu.ac.uk/ id/eprint/25768. Last accessed: 7 August 2021.

48. Public Health England. Effectiveness of social prescribing: an evidence synthesis. 2019. Available at: https://www.scie-socialcareonline. org.uk/effectiveness-of-socialprescribing-an-evidence-synthesis/r/ a116f00000Uhql9AAB. Last accessed: 7 August 2021

49. The Royal Australian College of General Practitioners (RACGP). Social prescribing roundtable, November 2019: report. 2019. Available at: https://www.racgp.org.au/FSDEDEV/ media/documents/RACGP/Advocacy/ Social-prescribing-report-andrecommendation.pdf. Last accessed: 7 August 2021

50. Bickerdike $L$ et al. Social prescribing: less rhetoric and more reality. A systematic review of the evidence. BMJ Open. 2017;7(4):e013384.

51. Osmanabad $V$ et al. Association between insulin resistance and the development of cardiovascular disease. Cardiovasc Diabetol. 2018;17(1):122.

52. Viscoli CM et al. Pioglitazone for secondary prevention after ischemic stroke and transient ischemic attack: rationale and design of the Insulin Resistance Intervention after Stroke trial. Am Heart J. 2014;168(6):823-9.

53. Viscoli CM et al. Pioglitazone after ischemic stroke or transient ischemic attack. N Engl J Med. 2016;375(7):704.

54. Yaghi S et al. Pioglitazone prevents stroke in patients with a recent transient ischemic attack or ischemic stroke. Circulation. 2018;137(5):455-63.

55. Ntaios G, Kent TA. Insulin Resistance
Intervention After Stroke trial of pioglitazone: is this perhaps the end of the beginning? Stroke. 2016;47(7):1962-4.

56. Yang $W$ et al. The effect of metformin on mortality and severity in COVID-19 patients with diabetes mellitus. Diabetes Res Clin Pract. 2021;178:108977.

57. Marques-Vidal $\mathrm{P}$ et al. Association between circulating cytokine levels, diabetes and insulin resistance in a population-based sample (CoLaus study). Clin Endocrinol (Oxf). 2013;78(2):232-41.

58. Marques-Vidal $\mathrm{P}$ et al. Association between inflammatory and obesity markers in a Swiss population-based sample (CoLaus study). Obes Facts. 2012;5(5):734-44.

59. Mehndiratta MM et al. Stroke in Asia: geographical variations and temporal trends. J Neurol Neurosurg Psychiatry. 2014;85(12):1308-12.

60. Bhaskar S et al. Trends in acute stroke presentations to an emergency department: implications for specific communities in accessing acute stroke care services. Postgrad Med J. 2019;95(1123):258-64.

61. Fernando, E. Cardiovascular disease in South Asian migrants. Can $J$ Cardiol. 2015;31(9):1139-50.

62. Adhikari R, Sanou D. Risk factors of diabetes in Canadian immigrants: a synthesis of recent literature. Can J Diabetes. 2012;36(3):142-50.

63. Tee LY et al. COVID-19 and undiagnosed pre-diabetes or diabetes mellitus among international migrant workers in Singapore. Front Public Health. 2020;8:584249.

64. Diabetes Prevention Program Research Group. The prevalence of retinopathy in impaired glucose tolerance and recent-onset diabetes in the Diabetes Prevention Program. Diabet Med. 2007;24(2):137-44.

65. The Diabetes Prevention Program Research Group. Design and methods for a clinical trial in the prevention of type 2 diabetes. Diabetes Care. 1999;22(4):623-34.

66. Wong TY et al. The prevalence and risk factors of retinal microvascular abnormalities in older persons: the Cardiovascular Health Study. Ophthalmology. 2003;110(4):658-66.
67. Nagi DK et al. Diabetic retinopathy assessed by fundus photography in Pima Indians with impaired glucose tolerance and NIDDM. Diabet Med. 1997;14(6):449-56.

68. Klein R et al. 15-year cumulative incidence and associated risk factors for retinopathy in nondiabetic persons. Arch Ophthalmol. 2010;128(12):1568-75.

69. Joachim $\mathrm{N}$ et al. The incidence and progression of age-related macular degeneration over 15 Years: the Blue Mountains Eye Study. Ophthalmology. 2015;122(12):2482-9.

70. Leibowitz HM et al. The Framingham Eye Study monograph: an ophthalmological and epidemiological study of cataract, glaucoma, diabetic retinopathy, macular degeneration, and visual acuity in a general population of 2631 adults, 1973-1975. Surv Ophthalmol. 1980;24(Suppl):335-610.

71. Svärdsudd K et al. Hypertensive eye ground changes. Prevalence, relation to blood pressure and prognostic importance. The study of men born in 1913. Acta Med Scand. 1978;204(3):159-67.

72. Palacio $\mathrm{S}$ et al. Lacunar strokes in patients with diabetes mellitus: risk factors, infarct location, and prognosis: the secondary prevention of small subcortical strokes study. Stroke. 1978:204(3):159-67.

73. Wu S et al. Glycated hemoglobin independently predicts stroke recurrence within one year after acute first-ever non-cardioembolic strokes onset in a Chinese cohort study. PLoS One. 2013;8(11):80690.

74. Bhaskar S et al. Key strategies for clinical management and improvement of healthcare services for cardiovascular disease and diabetes patients in the coronavirus (COVID-19) settings: recommendations from the REPROGRAM Consortium. Front Cardiovasc Med. 2020;7:112

75. Bhaskar S et al. Call for action to address equity and justice divide during COVID-19. Front Psychiatry. 2020;11:559905.

76. Venketasubramanian $\mathrm{N}$ et al. Stroke care during the COVID-19 pandemic: international expert panel review. Cerebrovasc Dis. 2021;50(3):245-61. 